\title{
PERFIL ESPIROMÉTRICO DE PACIENTES ATENDIDOS NO LABORATÓRIO DE FISIOTERAPIA CARDIORESPIRATÓRIA (LAFICRE)
}

\author{
SPIROMETRIC PROFILE OF PATIENTS TREATED AT \\ CARDIOPULMONARY PHYSIOTHERAPY LABORATORY (LAFICRE)
}

\begin{abstract}
Carolini Domingos ${ }^{1}$, Franciele Gonçalves França ${ }^{2}$, Ravelle da Silva Dell Osbel ${ }^{3}$, Lucas Vieira Machado ${ }^{4}$, Kristian Madeira ${ }^{5}$, Bruno Luiz da Silva Pieri ${ }^{6}$, Eduardo Ghisi

Victor $^{7}$
\end{abstract}

\begin{abstract}
Resumo
A espirometria é um exame que tem como objetivo auxiliar no diagnóstico, classificação e quantificação dos distúrbios ventilatórios (DV). A avaliação e determinação da gravidade é padronizada e de suma importância para detectar DV, acompanhar a evolução do paciente e dar o prognóstico de certas patologias. Objetivo: Analisar o perfil espirométrico de pacientes atendidos no Laboratório de Fisioterapia Cardiopulmonar (LAFICRE). Método: Trata-se de uma pesquisa de campo quantitativa, de abordagem retrospectiva com base nos laudos de espirometrias realizadas no LAFICRE nos anos de 2017/2018, onde foram analisados 256 laudos. Resultados e discussão: A partir da análise das espirometrias foi possível relacionar os resultados dos pacientes com o sexo, idade, profissão e IMC, e com isso perceber que o perfil do paciente está diretamente relacionado ao resultado dos exames. Conclusão: A partir destes resultados será possível propor melhorias ao atendimento oferecidos a comunidade e direcionando os atendimentos que ocorram em grupos para melhor atender a essa população.
\end{abstract}

Palavras-chave: Espirometria, Distúrbios, Ventilatórios.

\section{Abstract}

Spirometry is an examination that aims to assist in the diagnosis, classification and quantification of ventilatory disorders (DV). The evaluation and determination of severity is padronized and of paramount importance to detect (DV), monitor the evolution of the patient and give the prognosis of certain pathologies. Objective: To analyze the spirometric profile of patients treated at the Cardiopulmonary Physiotherapy Laboratory (LAFICRE). Method: This is a quantitative field research, a retrospective approach based on spirometry reports performed in LAFICRE in 2017/2018, where 256 reports were analyzed. Results: and discussion: From the

\footnotetext{
${ }^{1}$ Acadêmica de Fisioterapia / Universidade do Extremo Sul Catarinense

${ }^{2}$ Acadêmica de Fisioterapia / Universidade do Extremo Sul Catarinense

${ }^{3}$ Acadêmica de Fisioterapia / Universidade do Extremo Sul Catarinense

${ }^{4}$ Acadêmico de Matemática / Universidade do Extremo Sul Catarinense

${ }^{5}$ Docente no curso de Fisioterapia na Universidade do Extremo Sul Catarinense

${ }^{6}$ Docente no curso de Fisioterapia na Universidade do Extremo Sul Catarinense

${ }^{7}$ Docente no curso de Fisioterapia na Universidade do Extremo Sul Catarinense
}

Revista Inova Saúde, Criciúma, Vol. 12, n. 2,julho. 2021. ISSN 2317-2460 
analysis of spirometry it was possible to relate the results of patients with gender, age, profession and BMI, and with this realize that the patient's profile is directly related to the results of the tests. Conclusion: From these results it will be possible to propose improvements to the service offered to the community and directing the care that occurs in groups to better serve this population.

Keywords: Spirometry, Disorders, Ventilatory

\section{INTRODUÇÃO}

Espirometria é um exame que mostra à medida que o ar entra e sai dos pulmões, conhecida como prova de função pulmonar, e tem como objetivo auxiliar no diagnóstico, classificação e quantificação dos distúrbios ventilatórios (DV)1

Na realização da espirometria são necessárias algumas precauções para que o resultado seja verídico. A compreensão e colaboração do paciente é de suma importância. O examinador deve possuir conhecimento técnico, saber orientar o paciente durante o exame e dar os devidos comandos aos pacientes.

Fatores extrínsecos como a temperatura e a umidade do ar podem interferir no desempenho do paciente, e alterar o resultado. $O$ aparelho deve ser devidamente calibrado com uma certa frequência para garantir a segurança do resultado ${ }^{(2,3)}$

No exame o paciente deve usar um clipe nasal, que deve estar posicionado corretamente para que o ar não saia de forma inadequada pelo nariz e não interfira no resultado ${ }^{(4)}$. Na maioria dos casos o paciente deve ser orientado a suspender o uso de broncodilatadores para que o mesmo não altere os valores obtidos, essa recomendação só não é válida quando o objetivo do exame é avaliar a efetividade do tratamento ${ }^{(5)}$.

No laudo da espirometria é necessário seguir os valores de referências, porém os mesmos podem variar de acordo com o sexo (sendo até $20 \%$ a mais no sexo masculino), idade, estatura, raça, peso e fatores técnicos tais como a postura durante o exame, procedimento, tipo de programa usado, temperatura do ambiente (6)

$\mathrm{Na}$ espirometria podemos obter diversos tipos de resultados entre eles estão normais, distúrbio ventilatório restritivo (DVR), distúrbio ventilatório obstrutivo (DRO), distúrbio ventilatório inespecífico $(\mathrm{DVI})$ e distúrbio ventilatório misto (DVM) ${ }^{(7,8)}$.No DVR há uma diminuição dos volumes pulmonares, seu quantificador é associado ao valor da Capacidade Vital Forçada (CVF) e é caracterizado pela respiração rápida e curta, 
uma baixa reserva pulmonar que pode levar a taquipneia e ao aumento do trabalho respiratório, aumento de secreção, atelectasia e insuficiência respiratória que são características das doenças pulmonares restritivas ${ }^{(9)}$. O quantificador do DVO está associado ao valor do Volume Expiratório Forçado no primeiro segundo (VEF1), nesse tipo de DV podemos encontrar alterações anatômicas, inflamações crônicas ou infecções de repetição ${ }^{(10)}$. É considerado DVI aquele que não dispomos da Capacidade Pulmonar Total (CPT), tem diminuição da CVF e não tenha indicativos de um DVR. O DVI é comumente associado a obesidade pois a mesma impede o aumento da CPT $(12,8)$. É possível encontrarmos pacientes com a presença de obstrução e restrição juntos esses são denominados DVM ${ }^{(11}$.

A avaliação e determinação da gravidade é padronizada e de suma importância para alguns casos específicos como na Doença Pulmonar Obstrutiva Crônica (DPOC) (DVO) e Fibrose Pulmonar (DVR) pois ajuda nos prognósticos das mesmas (12).

A função da epidemiologia é a produção de evidências cientificas relevantes a Saúde Pública para isso visa usufruir dos conhecimentos adquiridos para o planejamento de intervenções, políticas e programas a partir dos resultados encontrados (13). Levando em consideração a importância da espirometria para detectar DV, acompanhar a evolução do paciente e dar o prognóstico de certas patologias, o presente estudo utilizou dados obtidos nesse exame para melhor compreender os resultados obtidos e com isso propor um melhor atendimento aos pacientes do LAFICRE, capacitando a equipe e melhorando o protocolo de atendimentos.

\section{MATERAIS E MÉTODOS}

Esse estudo trata-se de uma pesquisa de campo quantitativa, de abordagem retrospectiva com base nos laudos de espirometrias realizadas no LAFICRE nos anos de 2017/2018, teve como objetivo traçar um perfil espirométrico dos pacientes. Foi utilizada a área de estudo do LAFICRE, localizado nas Clínicas Integradas da Universidade do Extremo Sul Catarinense (UNESC). Foram avaliados nos laudos de espirometria, o sexo, idade cronológica, idade pulmonar, IMC e profissão, laudos que não portavam algumas dessas informações foram retirados da pesquisa. 
Os dados coletados foram analisados com auxílio do software IBM Statistical Package for the Social Sciencies (SPSS) versão 21.0 e Microsoft Office Excel 2010. As variáveis IMC, idade cronológica e idade pulmonar foram expressas por meio de média e desvio padrão pois seguiram distribuição Normal. As variáveis sexo, profissão, classificação do IMC e resultado da espirometria foram expressas por meio de frequência e porcentagem.

Os testes estatísticos foram realizados com um nível de significância $p<0,05$ e, portanto, confiança de $95 \%$. A distribuição dos dados quanto à normalidade foi avaliada por meio da aplicação do teste de Kolmogorov-Smirnov. A comparação da média de idade cronológica entre as categorias do resultado da espirometria foi empregada o teste $\mathrm{H}$ de Kruskal-Wallis seguido do post hoc teste de Dunn quando observada significância estatística. A correlação entre a idade cronologia e idade pulmonar foi realizada por meio do cálculo do coeficiente de correlação de Pearson e diagrama de dispersão. A investigação da existência de associação entre o resultado da espirometria e as variáveis sexo, profissão e IMC foi realizada por meio da aplicação do teste Razão de Verossimilhança, seguido de análise de resíduo quando observada significância estatística.

Esta pesquisa só teve início após submissão e aprovação pelo comitê de ética em pesquisa e humanos (CEP) da Universidade do Extremo Sul Catarinense - Unesc sob parecer $n^{0}=3.344 .733$.

\section{RESULTADOS}

Foram analisadas 258 espirometrias, realizadas entre o ano de 2017 e 2018, destas 137 (53,1\%) eram laudos de paciente do sexo feminino e 121 (46,9\%) do sexo masculino, a idade cronológica média dos pacientes que realizaram o exame foi de $54,41 \pm 17,40$. Em relação a profissão notou-se um grande número de aposentados, sendo $42,2 \%$ deles, seguido por do $\operatorname{lar}(15,1 \%)$ e estudante $(7,4 \%)$, uma subcategoria foi criada para mineiros $(1,6 \%)$ e mineiros aposentados $(1,2 \%)$, o restante das profissões foi agrupado em uma subcategoria denominada "outros" que resultaram em 32,6\%. Outro dado que se destacou foi o Índice de Massa Corporal (IMC), sendo que apenas $31 \%$ estavam dentro da normalidade segundo a Organização Mundial da 
Saúde, 26,4\% apresentavam pré-obesidade, 25,2\% obesidade grau I e 8,9\% obesidade grau II.

A idade cronológica média foi de 54,41 $\pm 17,40$, tendo diferença significativa quando comparada a idade pulmonar que foi de 71,62 $\pm 21,18$. Podemos observar melhor essa diferença na Figura 1.

Figura 1: Relação entre idade cronológica e idade pulmonar:

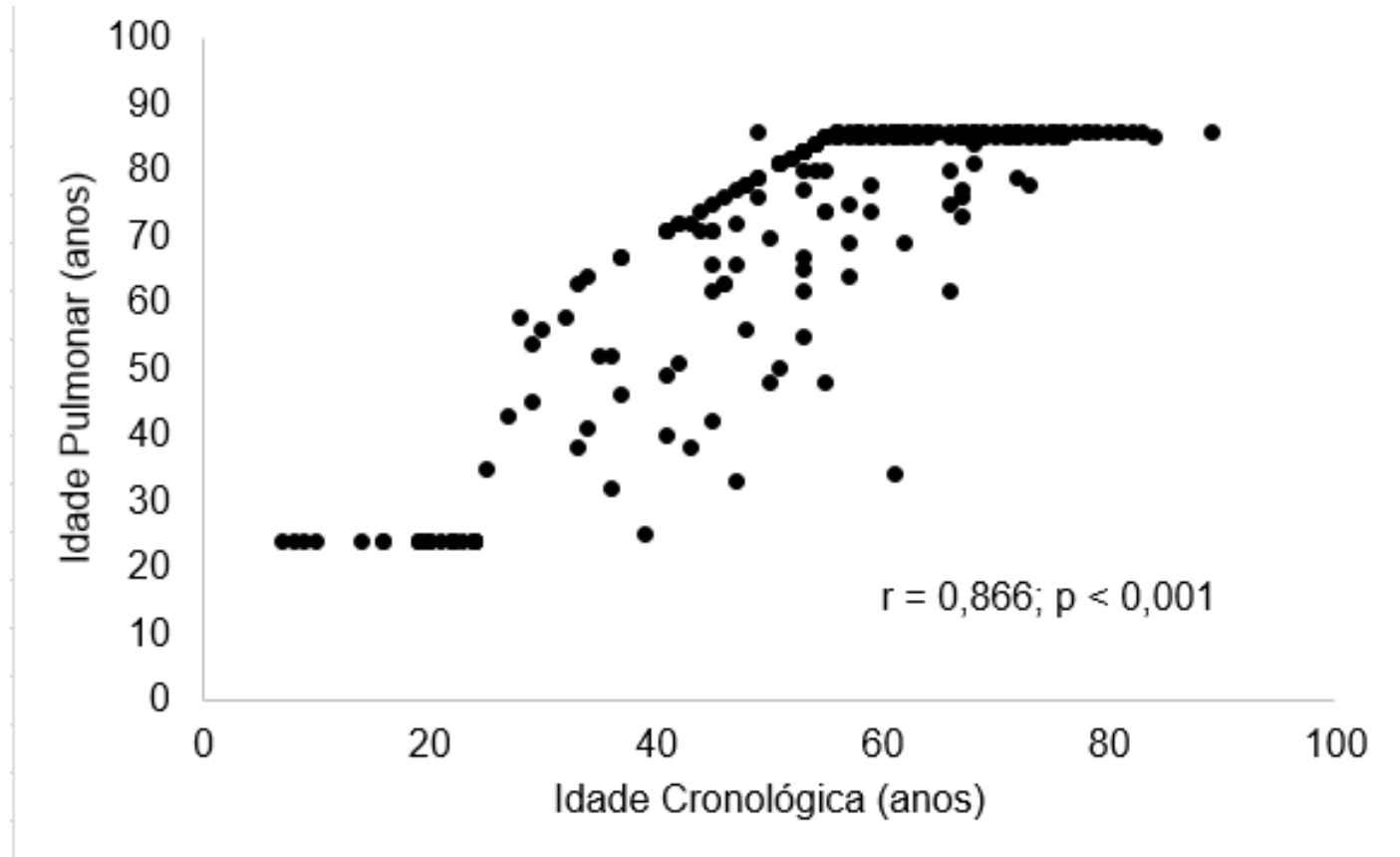

O exame mostra dois resultados, sendo o primeiro antes (Figura 2) e o segundo após o uso de broncodilatador (Figura 3), na base 36,0\% pacientes apresentaram DVR, 33,7\% DVO e 30,2\% deles não apresentaram alterações, já após o uso de broncodilatador o número de pacientes com DVR diminui em um caso, o DVO aumentou em 4 casos, e diminui 3 dos que estavam dentro dos valores de normalidade (Figura 2). 


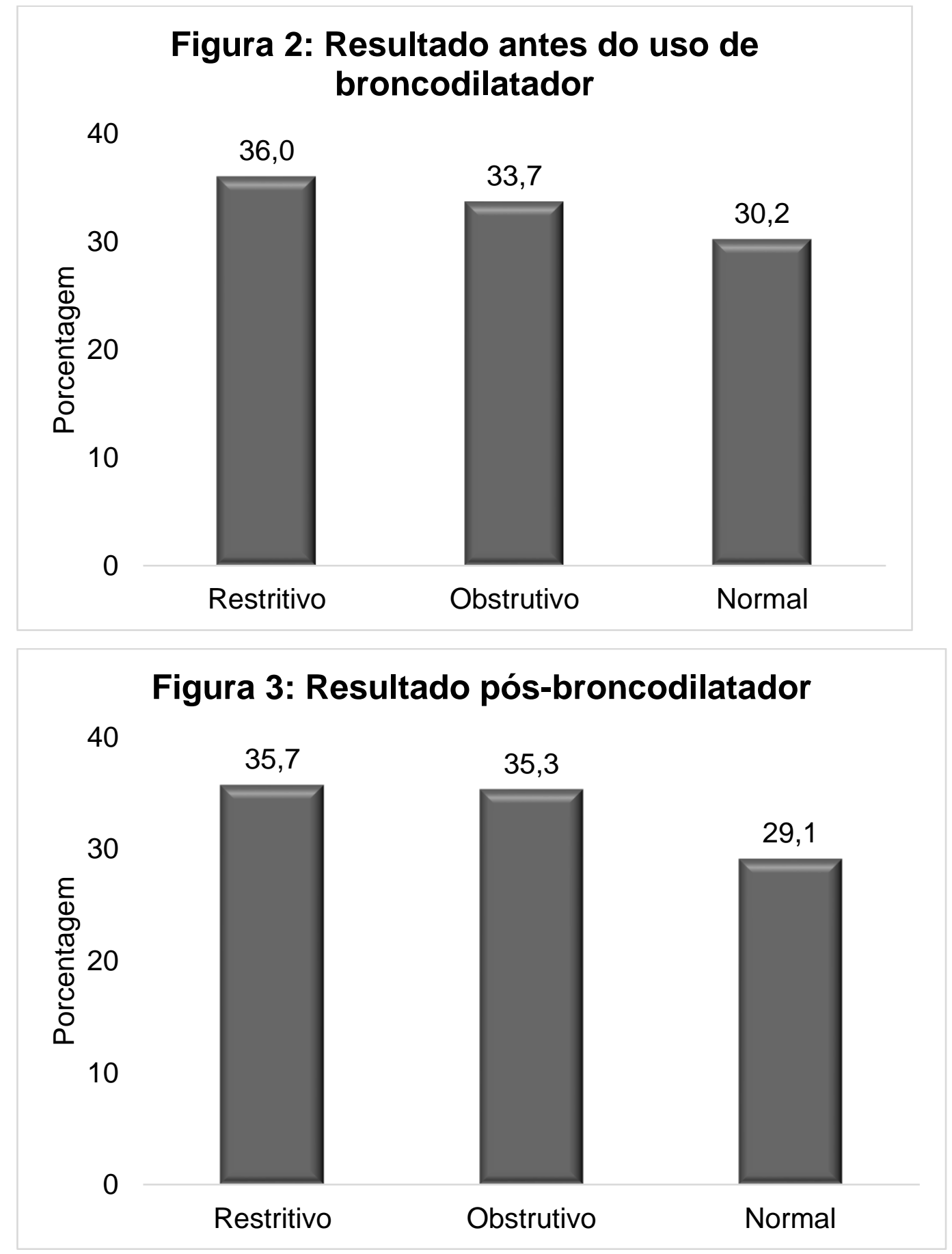

Dentre os resultados encontrados 92 pacientes apresentavam DVR, 91 DVO e 75 pacientes estavam dentro dos padrões de normalidade (Tabela 2). Com intuito de verificar a gravidade dos distúrbios ventilatórios, se destacaram os pacientes com restrição suave $(18,6 \%)$, restrição moderadamente severa $(14,7 \%)$, e obstrutivo muito severo e moderadamente severo $(10,1 \%)$, observamos esses valores de forma simplificada na Figura 4. 


\section{Figura 4: Resultado pós-broncodilatador}

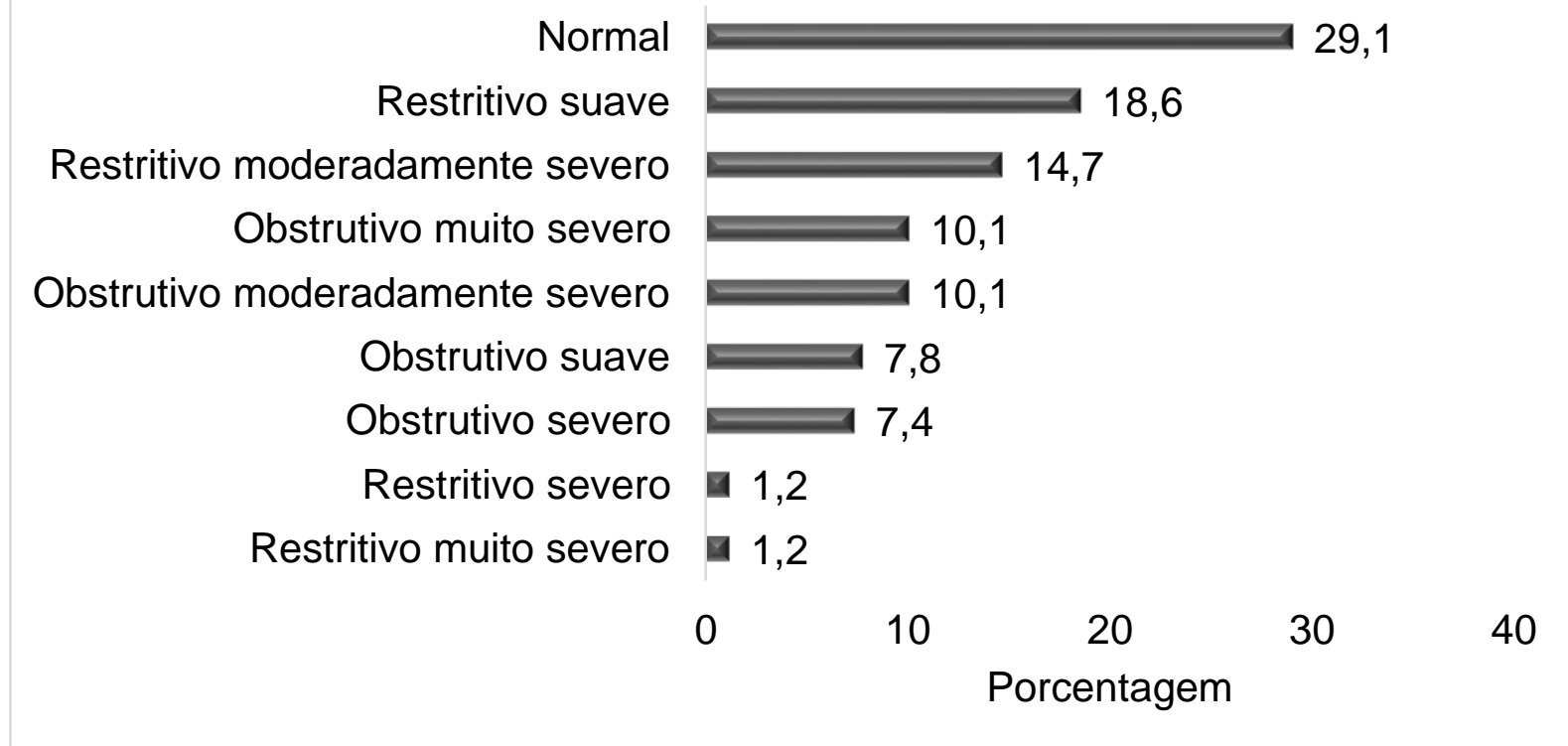

Foram relacionados os resultados obtidos nos testes espirométrico com o sexo, idade, profissão e IMC dos pacientes. Quando correlacionado os resultados dos testes com os sexos dos pacientes não houve diferença significativa, já com a média de idade, os laudos dentro dos parâmetros da normalidade obtiveram uma média de idade relativamente menor do que os que apresentaram algum distúrbio ventilatório. Houve um aumento gradativo da idade de acordo com a gravidade do distúrbio. Para correlacionar os resultados com as profissões foi necessário dividíei-las em categorias, sendo que os aposentados representaram a maioria, em relação a quase todos os DV, exceto nos DVR suave e muito severo que ocuparam o segundo lugar, dos pacientes sem DV a grande maioria estava na categoria de "outros", ou seja, relacionado aos pacientes ativos profissionalmente. Os mineiros e mineiros aposentados tiveram relação com DVO suave, DVO moderadamente severo e DVR moderadamente severo (Tabela 1). 
Tabela 3. Correlação entre os resultados obtidos nos testes espirométrico com o sexo, idade, profissão e IMC dos pacientes.

\begin{tabular}{|c|c|c|c|c|c|c|c|c|c|c|}
\hline & \multicolumn{9}{|c|}{ Resultado da Espirometria, $\mathbf{n}(\%)$, Média \pm DP } & \multirow[b]{2}{*}{ Valor-p } \\
\hline & $\begin{array}{c}0 \\
\mathrm{n}=75\end{array}$ & $\begin{array}{c}1 \\
n=20\end{array}$ & $\begin{array}{c}2 \\
n=26\end{array}$ & $\begin{array}{c}3 \\
n=19\end{array}$ & $\begin{array}{c}4 \\
n=26\end{array}$ & $\begin{array}{c}5 \\
n=48\end{array}$ & $\begin{array}{c}6 \\
n=38\end{array}$ & $\begin{array}{c}7 \\
n=3\end{array}$ & $\begin{array}{c}8 \\
n=3\end{array}$ & \\
\hline \multicolumn{11}{|l|}{ Sexo } \\
\hline Feminino & $48(64,0)$ & $8(40,0)$ & $11(42,3)$ & $10(52,6)$ & $9(34,6)$ & $31(64,6)$ & $17(44,7)$ & $1(33,3)$ & $2(66,7)$ & \multirow{2}{*}{$0,077^{\dagger}$} \\
\hline Masculino & $27(36,0)$ & $12(60,0)$ & $15(57,7)$ & $9(47,4)$ & $17(65,4)$ & $17(35,4)$ & $21(55,3)$ & $2(66,7)$ & $1(33,3)$ & \\
\hline \multicolumn{11}{|l|}{ Profissão } \\
\hline Aposentado & $12(16,0)$ & $9(45,0)$ & $12(46,2)$ & $14(73,7)^{c}$ & $19(73,1)^{c}$ & $16(33,3)$ & $24(63,2)^{c}$ & $2(66,7)$ & $1(33,3)$ & \multirow[t]{6}{*}{$<0,001^{\dagger}$} \\
\hline Mineiro* & $1(1,3)$ & $0(0,0)$ & $1(3,8)$ & $0(0,0)$ & $0(0,0)$ & $0(0,0)$ & $1(2,6)$ & $0(0,0)$ & $0(0,0)$ & \\
\hline Do lar & $16(21,3)$ & $2(10,0)$ & $2(7,7)$ & $3(15,8)$ & $1(3,8)$ & $9(18,8)$ & $4(10,5)$ & $1(33,3)$ & $1(33,3)$ & \\
\hline Mineiro & $2(2,7)$ & $1(5,0)$ & $1(3,8)$ & $0(0,0)$ & $0(0,0)$ & $0(0,0)$ & $0(0,0)$ & $0(0,0)$ & $0(0,0)$ & \\
\hline Estudante & $13(17,3)^{\mathrm{c}}$ & $3(15,0)$ & $0(0,0)$ & $0(0,0)$ & $0(0,0)$ & $3(6,3)$ & $0(0,0)$ & $0(0,0)$ & $0(0,0)$ & \\
\hline Outros & $31(41,3)$ & $5(25,0)$ & $10(38,5)$ & $2(10,5)$ & $6(23,1)$ & $20(41,7)$ & $9(23,7)$ & $0(0,0)$ & $1(33,3)$ & \\
\hline Idade (anos) & $44,48 \pm 19,97^{\text {a }}$ & $55,60 \pm 20,89$ & $55,69 \pm 12,12$ & $65,16 \pm 7,46^{b}$ & $63,27 \pm 13,00^{b}$ & $53,44 \pm 15,08$ & $61,26 \pm 11,89^{b}$ & $65,67 \pm 16,17$ & $65,00 \pm 14,93$ & $<0,001^{\ddagger}$ \\
\hline \multicolumn{11}{|c|}{ IMC } \\
\hline $\begin{array}{l}\text { Magreza } \\
\text { grau III }\end{array}$ & $3(4,0)$ & $0(0,0)$ & $0(0,0)$ & $0(0,0)$ & $0(0,0)$ & $0(0,0)$ & $0(0,0)$ & $0(0,0)$ & $0(0,0)$ & \multirow[t]{8}{*}{$0,427^{\dagger}$} \\
\hline Magreza & $1(1,3)$ & $0(0,0)$ & $0(0,0)$ & $0(0,0)$ & $0(0,0)$ & $0(0,0)$ & $0(0,0)$ & $0(0,0)$ & $0(0,0)$ & \\
\hline $\begin{array}{l}\text { grau II } \\
\text { Magreza } \\
\text { grau I }\end{array}$ & $3(4,0)$ & $0(0,0)$ & $2(7,7)$ & $0(0,0)$ & $1(3,8)$ & $0(0,0)$ & $1(2,6)$ & $0(0,0)$ & $0(0,0)$ & \\
\hline Eutrofia & $27(36,0)$ & $9(45,0)$ & $11(42,3)$ & $5(26,3)$ & $6(23,1)$ & $12(25,0)$ & $8(21,1)$ & $1(33,3)$ & $1(33,3)$ & \\
\hline $\begin{array}{l}\text { Pré- } \\
\text { obesidade }\end{array}$ & $17(22,7)$ & $5(25,0)$ & $5(19,2)$ & $7(36,8)$ & $10(38,5)$ & $15(31,3)$ & $7(18,4)$ & $0(0,0)$ & $2(66,7)$ & \\
\hline $\begin{array}{l}\text { Obesidade } \\
\text { grau I }\end{array}$ & $17(22,7)$ & $6(30,0)$ & $7(26,9)$ & $3(15,8)$ & $6(23,1)$ & $11(22,9)$ & $14(36,8)$ & $1(33,3)$ & $0(0,0)$ & \\
\hline Obesidade & $4(5,3)$ & $0(0,0)$ & $1(3,8)$ & $4(21,1)$ & $1(3,8)$ & $8(16,7)$ & $5(13,2)$ & $0(0,0)$ & $0(0,0)$ & \\
\hline $\begin{array}{l}\text { grau II } \\
\text { Obesidade } \\
\text { grau III }\end{array}$ & $3(4,0)$ & $0(0,0)$ & $0(0,0)$ & $0(0,0)$ & $2(7,7)$ & $2(4,2)$ & $3(7,9)$ & $1(33,3)$ & $0(0,0)$ & \\
\hline
\end{tabular}

A relação entre os DV e o IMC dos pacientes não apresentou resultados significativos, porém alguns dados interessantes foram observados (Figura 5). Os pacientes abaixo do peso apresentavam laudos com parâmetros dentro da normalidade. Já os pacientes com sobrepeso apresentavam alterações como, DVO Severo $36,8 \%$ foram classificados com pré-obesidade e $22,1 \%$ com obesidade grau II, nos DVO muito severo $38,5 \%$ estavam em pré-obesidade e $23,1 \%$ com obesidade grau I, já os pacientes com DVR moderadamente severo $36,8 \%$ era obeso grau I e no DVR severo 33,3\% foram classificados com pré-obesidade igualmente distribuído com

\section{Figura 5: IMC}

Magreza grau III 1,2

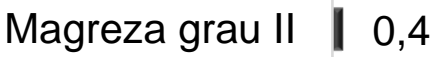

Magreza grau I $\square$ 2,7

Eutrofia

31,0

Pré-obesidade

26,4

Obesidade grau I

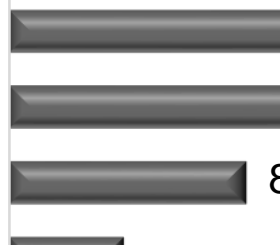

25,2

Obesidade grau II

\section{8,9}

Obesidade grau III 4,3

$0 \quad 10$

20

30

40

Revista Inova Saúde, Criciúma, Vol. 12, n. 2,julho. 2021. ISSN 2317-2460 
$33,3 \%$ eram obesos grau I, já os DVR muito severo $66,7 \%$ estavam com préobesidade (Figura 6).

Figura 6: Resultado da Espirometria X classificação IMC
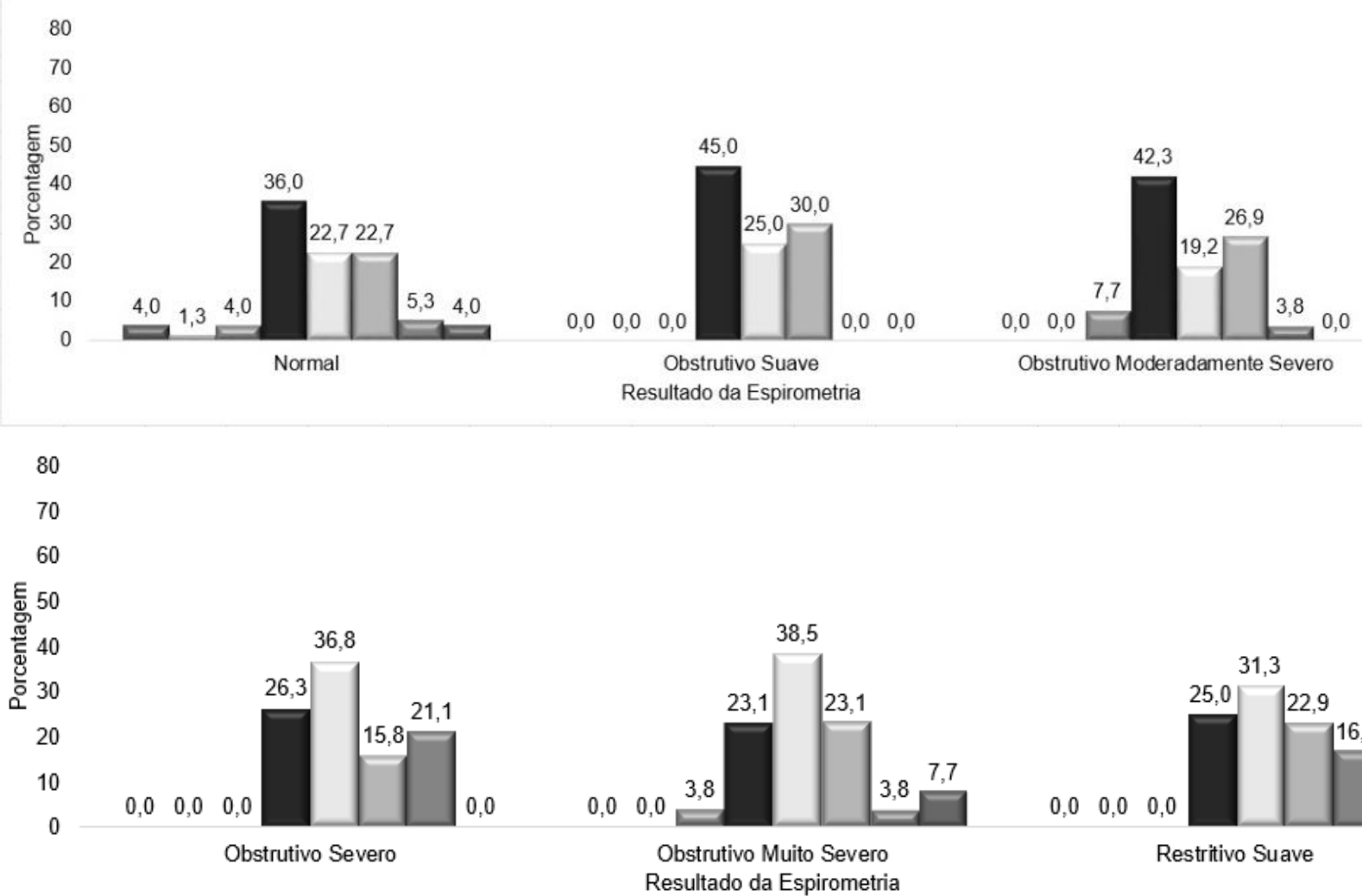

$\begin{array}{lll}0,0 & 0,0 & 0,0\end{array}$

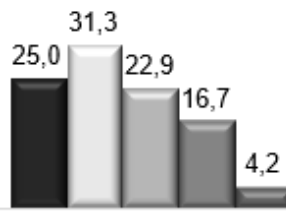

Resultado da Espirometria

Restritivo Suave

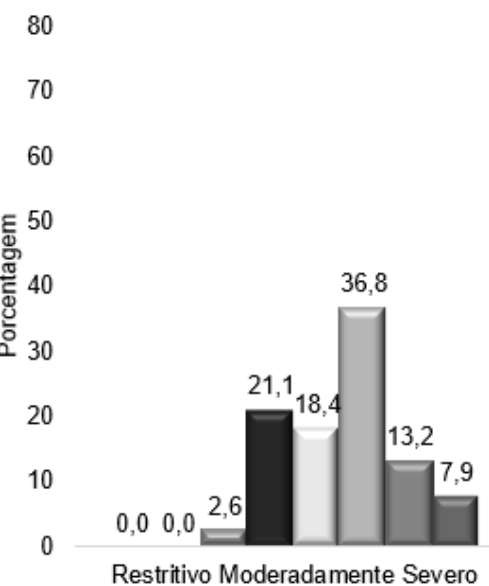

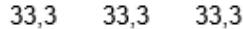

Restritivo Severo

Resultado da Espirometria
Classificação IMC

- Magreza grau III

a Magreza grau II

- Magreza grau I

Eutrofia

$\square$ Pré-obesidade

a Obesidade grau

abesidade grau II

a Obesidade grau III

$\begin{array}{llll}0,0 & 0,0 & 0,0\end{array} \quad \begin{array}{llll}0,0 & 0,0 & 0,0\end{array}$

Restritivo Muito Severo

$p=0,427$

\section{DISCUSSÃO}

Nos resultados do presente estudo foi constatado que a procura do exame é igualmente distribuída entre os sexos, segundo Carneiro(14), mesmo os homens 
apresentam uma maior taxa de mortalidade e uma expectativa de vida menor, e não procuram os serviços preventivo(14). Em relação ao cuidado com a saúde e melhora da qualidade de vida, as mulheres que realizam exames preventivo e procuram serviço de saúde. No presente estudo, os laudos considerados normais foram predominantemente de mulheres, correlacionado com a teoria, em que o sexo feminino procura mais o serviço de saúde com intuito de prevenção.

No estudo foi verificado a idade elevada dos pacientes e uma grande parte deles já aposentado, evidenciando a relação entre cuidado com a saúde e o envelhecimento. De acordo com Nicolato e colaboradores ${ }^{(15)}$, idosos independentes são os que mais procuram atendimento a nível ambulatorial. Assim como o estudo de Patrocínio e colaboradores ${ }^{(16)}$ que relacionou a faixa etária elevada com a maior facilidade dessa população a acessos de serviços de saúde, o que justifica a idade elevada dos pacientes desse estudo.

Dos laudos analisados, 127 pacientes apresentaram algum grau de obesidade, segundo a literatura a obesidade tem relação direta com a mecânica pulmonar, relacionada com a dificuldade da expansibilidade do tórax, alteração na posição do diafragma, como a redução e o deslocamento do mesmo, e a redução capacidade residual funcional (CRF) o que leva as alterações nas características de pressãovolume do tórax. Outra alteração causada pelo IMC elevado é a redução do volume de reserva expiratório (VRE) correlacionado com a menor mobilidade e mecânica diafragma no tórax que acaba sobrecarregando os músculos acessórios ${ }^{(17)}$. Podemos associar a obesidade com algumas complicações respiratórias como: Asma, DPOC, embolia pulmonar, pneumonia aspirativa e síndrome da hiperventilação da obesidade $(18,19)$.

D.'Ávila ${ }^{(20)}$ analisou a idade pulmonar em obesos mórbidos e observou um aumento na idade pulmonar em relação a idade cronológica em torno de 10 anos, constatou também que quanto maior o IMC, maior a idade pulmonar, o mesmo fator é visualizado em estudos que avaliaram a função pulmonar em indivíduos tabagista ${ }^{(21,22)}$, já em estudos que analisaram pacientes considerados saudáveis, não tabagistas e com IMC normal não se observou diferença significativa entre as idade cronológica e a idade pulmonar ${ }^{(23)}$. Isso nos leva a acreditar que o diagnóstico da idade pulmonar se torna mais fácil de compreender a gravidade de uma patologia. 
A espirometria é o principal exame para o diagnóstico de DVO, para mensurar sua gravidade e para acompanhar a evolução funcional e eficácia do tratamento escolhido, porém segundo o mesmo, uma espirometria com laudo normal não necessariamente descarta o DVO(11). Uma doença com obstrução ao fluxo aéreo pode ter como resultado um padrão restritivo na espirometria para isso pode ser solicitado outras formas de avaliação para obter um resultado fidedigno( ${ }^{(24)}$. Pode ser sugerido um DVR a partir de uma espirometria quando ocorre redução significativa da Capacidade Vital (CV) associada a normalidade ou aumento da relação VEF1/CVF, porém o diagnóstico de DVR, não pode ser afirmado apenas pela CV (25).

A espirometria apresenta limitações quando se trata dos valores de referências de acordo com as faixas etárias extremas, porém ainda é o principal exame para verificar a funcionalidade do sistema respiratório, e provavelmente continuará sendo o mais empregado, principalmente como forma de teste diagnóstico funcional inicial (26).

\section{CONCLUSÃO}

Podemos concluir com o presente estudo que apesar das dificuldades encontradas no teste de função pulmonar, principalmente quando se trata de um DVR, o mesmo é considerado padrão ouro para os DVO, levando em consideração que é a espirometria é um exame de fácil acesso, e fácil execução a mesma é extremamente válida tanto para auxiliar no diagnóstico, classificação e quantificação dos DV, tanto quanto para acompanhar a evolução do paciente e dar o prognóstico do mesmo.

Um diagnóstico fidedigno depende muito da capacitação do examinador para que o mesmo possua conhecimento técnico e saiba explicar o exame e dar os devidos comandos ao paciente, com isso obtemos um resultado mais verídico. Este estudo nos possibilita fazer uma reflexão sobre os melhores protocolos adotados pelos acadêmicos que atendem no LAFICRE, atuando no processo de capacitação e conhecimento sobre o perfil dos pacientes e tratamentos mais efetivo voltado ao público que participa dos grupos. 


\section{AGRADECIMENTOS}

Nosso agradecimento a todos que de alguma forma contribuíram para este trabalho acontecer, porém em especial ao Laboratório de Fisioterapia Cardiorrespiratória (LAFICRE) por ter aceitado nossa proposta e sempre estar à disposição para nossas dúvidas.

\section{REFERÊNCIAS}

1. Sociedade Brasileira de Pneumologia e Tisiologia. IV Diretrizes Brasileiras para o Manejo da Asma. J Bras Pneumol. 2006.

2. De Castro Pereira, Carlos Alberto; SATO, Taeko; Rodrigues, Sílvia Carla. Novos valores de referência para espirometria forçada em brasileiros adultos de raça branca. J Bras Pneumol, v. 33, n. 4, p. 397-406, 2007.

3. Oliveira, Luiz Fernando. Avaliação dos parâmetros sanguíneos e ventilatórios de trabalhadores rurais expostos a agrotóxicos. 2018.

4. Da Silva, Cássio Magalhães et al. Comparação da função pulmonar no pré e pós-operatório de pacientes submetidos à cirurgia cardíaca aberta de revascularização do miocárdio de um hospital filantrópico de Feira de Santana/BA. Fisioterapia Brasil, v. 14, n. 1, 2018.

5. De Lima Azambuja, Renato; Lopes, Agnaldo José. Orientações para os testes de função pulmonar: espirometria. Pulmão RJ, v. 27, n. 1, p. 5-10, 2018.

6. Drumond, S. C., Fernandes Fontes, M. J., De Assis, I., Duarte, M. A., Lamounier, J. A., Orlandi, L., ... \& Rodrigues Machado, . Comparação entre três equações de referência para a espirometria em crianças e adolescentes com diferentes índices de massa corpórea. Jornal Brasileiro de Pneumologia, v. 35, n. 5, 2009.

7. Rodrigues, M. T., Molinari, D. F., Barreto, M., Saldanha, S., \& Fiterman, J. O papel da razão $\mathrm{FEF} 50 \% / 0,5 \mathrm{cVF}$ no diagnóstico dos distúrbios ventilatórios obstrutivos. Jornal brasileiro de pneumologia. Brasilia. Vol. 36, n. 1 (jan. 2010), p. 44-50, 2010.

8. Silveira, Keller Guimarães. A influência do posicionamento corporal sobre a prova de função pulmonar em indivíduos adultos saudáveis. 2018. 
9. Wooten III, WI, Muenzer, J., Vaughn, BV, \& Muhlebach, MS (2013). Relationship of sleep to pulmonary function in mucopolysaccharidosis II. The Journal of pediatrics, v. 162, n. 6, p. 1210-1215, 2013.

10. de Oliveira, J. L., Camelier, F. W. R., Jesus, F. R., Barreto, R. M. A., Ferreira, P. P., Terse-Ramos, R., ... \& Mendes, C. M. C. Perfil ventilatório e capacidade funcional de pacientes com mucopolissacaridoses. Fisioterapia Brasil, v. 18, n. 1, p. 69-79, 2017.

11. Rufino, Rogério; Da Costa, Cláudia Henrique; LOPES, Agnaldo José. Diagnóstico e classificação do distúrbio ventilatório obstrutivo. Pulmão RJ, v. 27, n. 1, p. 81-88, 2018.

12. Rubin, Asalberto Sperb. Espirometria na prática médica Spirometry in medical practice. Revista AMRIGS, v. 49, n. 3, p. 183-194, 2005.

13. (Szkio, M. (2015). Epidemiologia translacional : algumas considerações. Epidemiologia e Serviços de Saúde, 24, 161-172.)

14. Carneiro, Viviane Santos Mendes; Adjuto, Raphael Neiva Praça; Alves, Kelly Aparecida Palma. Saúde do homem: identificação e análise dos fatores relacionados à procura, ou não, dos serviços de atenção primária. Arquivos de Ciências da Saúde da UNIPAR, 2019, 23.1.

15. Nicolato, Fernanda Vieira; Do Couto, Alcimar Marcelo; De Castro, Edna Aparecida Barbosa. Capacidade de autocuidado de idosos atendidos pela consulta de enfermagem na atenção secundária à saúde. Revista de Enfermagem do Centro Oeste Mineiro, 2016, 6.2.

16. Patrocínio, D. A., Júnior, V. J., de Abreu Rodrigues, F., Vinha, P. P., \& Bagatella, L.Espirometria no idoso: estudo retrospectivo de 438 casos. Revista $\begin{array}{llll}\text { de Ciências } & \text { Médicas, }\end{array}$ (https://www.revistas.usp.br/fpusp/article/view/78398).

17. Melo, V. A. D. Asma, idade pulmonar e tipo de internação em pacientes com obesidade submetidos à cirurgia bariátrica por vídeolaparoscopia. 2011.

18. Beuther, David A.; Sutherland, E. Rand. Overweight, obesity, and incident asthma: a meta-analysis of prospective epidemiologic studies. American journal of respiratory and critical care medicine, v. 175, n. 7, p. 661-666, 2007. 
19. Tavares, M. G., Nascimento, A. C. S. D., Ferraz, M. C. C. N., Medeiros, R. A. B. D., Cabral, P. C., \& Burgos, M. G. P. D. A. Excesso de peso e obesidade em portadores de doença pulmonar obstrutiva crônica. Braspen J, p. 58-62, 2017.

20. Melo, S. M. D. Á., de Melo, V. A., de Melo, E. V., de Menezes Filho, R. S., de Castro, V. L., \& Barreto, M. S. P. Envelhecimento pulmonar acelerado em pacientes com obesidade mórbida. Jornal Brasileiro de Pneumologia, 2010, 36.6: $746-752$.

21. da Silva, L. C. C., de Araújo, A. J., de Queiroz, Â. M. D., Sales, M. D. P. U., \& de Oliveira Castellano, M. V. C. Controle do tabagismo: desafios e conquistas. Jornal Brasileiro de Pneumologia, 2016, 42.4: 290-298.

22. Parkes, G., Greenhalgh, T., Griffin, M., \& Dent, R. Effect on smoking quit rate of telling patients their lung age: the Step2quit randomised controlled trial. Bmj, 2008, 336.7644: 598-600.

23. Morris, James F.; Temple, William. Spirometric "lung age" estimation for motivating smoking cessation. Preventive medicine, 1985, 14.5: 655-662

24. Schultz, K., D'Aquino, L. C., Soares, M. R., Gimenez, A., \& de Castro Pereira, C. AVolumes pulmonares e resistência das vias aéreas em pacientes com possível padrão restritivo à espirometria. Jornal Brasileiro de Pneumologia, 2016, 42.5: 341-347.

25. Sociedade Brasileira de Pneumologia e Tisiologia, \& Sociedade Brasileira de Pneumologia e Tisiologia. (2002). Diretrizes para testes de função pulmonar. $J$ pneumol, 28(Suppl 3), S1-S238.

26. Trindade, Alexandre Moreto; Souza, Thiago Lins Fagundes de; Albuquerque, André Luís Pereira. A interpretação da espirometria na prática pneumológica: até onde podemos avançar com o uso dos seus parâmetros. pulmão RJ, 2015, 24.1:3-7. 